\title{
El enfoque diferencial étnico y el deber de la consulta previa en el acuerdo de paz
}

\author{
Recibido: 10 de junio de 2019 • Aprobado: 16 de octubre de 2019 \\ https://doi.org/10.22395/ojum.v19n38al1
}

\author{
Isabela Figueroa** \\ Pedro José Palacio Pardo*** \\ Ariel Fernando Amado Carreño****
}

\section{RESUMEN}

El artículo reflexiona sobre la noción de enfoque diferencial territorial y étnico contenido en el acuerdo de paz entre el Estado colombiano y las FARC. Se busca identificar los efectos jurídicos que tiene esta noción y de qué manera su introducción en el acuerdo de paz ha traído una mayor protección de los derechos de los pueblos indígenas y otras minorías étnicas. Dado que el acuerdo reconoce que la herramienta jurídica para la realización de la paz en las tierras y territorios de las minorías étnicas es la consulta previa, también se hace un análisis preliminar de los efectos de esta propuesta de ley. Actualmente se discute en el Poder Legislativo lo que esta consulta que puede traer para la implementación del acuerdo.

Palabras clave: enfoque diferencial; acuerdos de paz; consulta previa; minorías étnicas.

* Ese artículo es resultado parcial del proyecto de investigación Alcance del enfoque diferencial en la implementación de los acuerdos de paz de Colombia en torno a la garantía de los derechos colectivos de las comunidades étnicas, apoyado por la Vicerrectoría de Investigación de la Universidad del Magdalena entre julio de 2017 a noviembre de 2019.

** Abogada con experiencia de trabajo junto a pueblos indígenas y comunidades tradicionales en diversos países de América, magíster en Derecho, magíster en Derecho y Política de Pueblos Indígenas, magíster en Derecho y doctora en Estudios Culturales Latinoamericanos. Profesora, Facultad de Humanidades, Universidad del Magdalena, Santa Marta, Colombia. Correo electrónico: ifigueroa@unimagdalena.edu. co, Orcid: https://orcid.org/0000-0002-9119-3615

$* *$ Estudiante de Derecho, semillerista del Grupo de Investigación Saberes jurídicos, Universidad del Magdalena (Grisjum) en la línea "Nuevas Perspectivas de los Derechos". Correo electrónico: palaciopedro08@ gmail.com, Orcid: https://orcid.org/0000-0003-4488-7507

**** Estudiante de Antropología, semillerista del Grupo de Investigación Saberes jurídicos, Universidad del Magdalena (Grisjum) en la línea "Nuevas Perspectivas de los Derechos". Correo electrónico: fernando. amadoc@gmail.com, Orcid: https://orcid.org/0000-0002-4166-3666 


\title{
The Ethnically Differentiated Approach and the Duty of the Prior Consultation in the Peace Agreement
}

\begin{abstract}
This article reflects around the ethnically differentiated approach contained within the peace agreement between the Colombian State and the former guerrilla group FARC. We aim to identify the judicial effects of this notion and in which way its introduction in the peace agreement has brought greater protection for the indigenous people and other ethnic minorities. Given that the agreement recognizes that the judicial tool for the development of peace in the lands and territories of the ethnic minorities is the prior consultation, we also perform a preliminary analysis of the effects of this law proposal. We are currently discussing in the Legislative Power what this consultation might bring for the implementation of the agreement.
\end{abstract}

Keywords: differentiated approach; peace agreements; prior consultation; ethnic minorities.

\section{A abordagem diferencial étnica e o dever da consulta prévia no acordo de paz}

\section{RESUMO}

Este artigo reflete sobre a noção de abordagem diferencial territorial e étnica contida no acordo de paz entre o Estado colombiano e as Forças Armadas Revolucionárias da Colômbia-Exército do Povo. Pretende-se identificar os efeitos jurídicos que essa noção tem e de que forma sua introdução no acordo de paz tem trazido uma maior proteção dos direitos dos povos indígenas e de outras minorias étnicas. Tendo em vista que o acordo reconhece que a consulta prévia é a ferramenta jurídica para realizar a paz nas terras e territórios das minorias étnicas, é realizada uma análise preliminar dos efeitos dessa proposta de lei. Na atualidade, é discutido, no Poder Legislativo, o que essa consulta pode trazer para a implementação do acordo.

Palavras-chave: abordagem diferencial; acordos de paz; consulta prévia; minorias étnicas. 


\section{INTRODUCCIÓN}

La firma del Acuerdo Final para la Terminación del Conflicto y la Construcción de una Paz Estable y Duradera, suscrito entre el gobierno colombiano y las FARC-EP en el año 2016, trajo a las poblaciones rurales de Colombia una serie de inquietudes relacionadas con el conflicto armado, o más bien, con su anunciado término: ¿Qué significa paz desde las políticas gubernamentales? ¿De qué manera esa perspectiva afecta a los habitantes del campo colombiano?

En el marco de los trabajos preparatorios para la elaboración de la Declaración sobre los Derechos de los Pueblos a la Paz, el Comité Asesor del Consejo de Derechos Humanos de ese organismo propone una concepción de paz que va más allá de la ausencia de violencia organizada, considerando especialmente "la protección amplia y efectiva de los derechos humanos, la igualdad de género y la justicia social, el bienestar económico y la expresión libre y generalizada de diferentes valores culturales, sin discriminación ni restricciones" (Asamblea General de Naciones Unidas, 2011, p. 5).

Según la Asamblea General de Naciones Unidas (2011) "el medio ambiente es un aspecto fundamental del derecho a la paz" (p. 17), y esta es una oportunidad para el medio ambiente en Colombia (Perald, 2016). Entre 1990 y 2013, alrededor del 58 \% de la deforestación en Colombia ocurrió en áreas afectadas por el conflicto armado. Además, una serie de derrames de crudo ha dejado un saldo de 4,1 millones de barriles derramados al ambiente, lo que ha sido dieciséis veces mayor al desastre de Exxon Valdez (Bustos y Jaramillo, 2016). Eso sin mencionar otros problemas ambientales, cuyos datos oficiales son difíciles de estimar, como la minería ilegal, los cultivos ilícitos o las minas antipersonas. En todo caso, el Gobierno colombiano estimó que la firma del acuerdo de paz podría ahorrar hasta 2,2 mil millones de dólares al año en daños medioambientales (Gaviria, 2016).

Las regiones más afectadas por el conflicto son las que tienen más necesidades básicas insatisfechas, y se espera que la firma de la paz traiga nuevos horizontes de desarrollo a estos lugares. Sin embargo, algunos sectores prevén que la firma del acuerdo de paz abrirá una gran porción de los bosques colombianos a la inversión extranjera, ya fuertemente presente en Colombia y en otras regiones de América Latina, especialmente en lo que atañe a las actividades mineras y las agroindustrias de monocultivos. Por lo tanto, sin una implementación cuidadosa de las medidas de apertura, es posible que se dé tal aumento de conflictos socioambientales que Colombia se vuelva uno de los países con más conflictos de esta índole en Latinoamérica (Semana, 2014). Esto iría, evidentemente, en un movimiento simétricamente opuesto a los objetivos del acuerdo de paz.

Para que los conflictos alrededor de la exploración de los recursos naturales y poblaciones locales no sean exacerbados, es fundamental la construcción de una relación 
de confianza entre los distintos sectores del Estado y las poblaciones históricamente afectadas por la guerra. El acuerdo prevé que la construcción y consolidación de la paz requiere el surgimiento de "nuevas fuerzas en el escenario político para enriquecer el debate y la deliberación alrededor de los grandes problemas nacionales" (Presidencia de la República, 2016, p. 7) que fortalezcan el pluralismo con el fin de contar con la representación de diferentes visiones e intereses sociales en el escenario político.

Una de las herramientas jurídicas para garantizar la representación de las distintas visiones e intereses de los pueblos indígenas en las decisiones del Gobierno colombiano es la consulta previa, prevista en el ordenamiento jurídico desde 1991 (Vallejo, 2016). Sin embargo, hasta el momento — y pese a que la Corte Constitucional ha fallado en múltiples ocasiones de manera favorable a los grupos étnicos-Colombia aún no cuenta con una legislación que defina un procedimiento de consulta adecuado a los parámetros internacionales de los derechos humanos. Y no solo eso, sino que la última propuesta de ley relativa al tema que se radicó en el Senado de la República ${ }^{1}$ no contaba con la aprobación de las organizaciones indígenas (Gaceta del Congreso 739 , 2018). La Organización Internacional del Trabajo ha identificado una serie de obstáculos en la implementación efectiva de la consulta previa en Colombia, entre ellos está la desconfianza mutua, por lo que emitió una serie de recomendaciones. Enfatizó que es necesaria la "construcción de una política pública de relacionamiento con los pueblos indígenas y los grupos étnicamente diferenciados" (OIT, 2016, pp. 58, 70). Esta desconfianza ha sido reconocida por las partes en el acuerdo de paz, especialmente en aquellos territorios que fueron más afectados por el conflicto (Presidencia de la República, 2016, p. 7).

En este texto, elaborado a partir de una visión interdisciplinar entre el Derecho y la Antropología, interesa identificar el alcance jurídico del enfoque diferencial étnico establecido en el capítulo étnico del acuerdo de paz y de qué manera este enfoque efectivamente protege a dichos grupos. Se indagó si la perspectiva diferenciada contenida en el acuerdo puede ofrecer una protección según los estándares del Sistema Internacional de Protección de los Derechos Humanos que permita la construcción de una paz que incluya la perspectiva de los grupos étnicos afectados por la guerra.

La revisión bibliográfica, jurisprudencial y legislativa sobre el acuerdo de paz, la consulta y el enfoque diferencial, así como las entrevistas semiestructuradas a intelectuales y autoridades indígenas, fueron los métodos utilizados para recoger la información que construye el análisis plasmado en este trabajo. Fueron entrevistados intelectuales y líderes de la comunidad afrodescendiente del corregimiento de San Juan de Palos Prietos (municipio de Puebloviejo) en el Magdalena y asesores indígenas que trabajan en la Comisión de la Verdad.

El proyecto de la Ley 134 de 2018 fue presentado en el Senado de Colombia el día 11 de septiembre de 2018 y archivado por haber sido radicado como proyecto de ley ordinaria, mientras que por tratar de la regulación de un derecho fundamental debió haber sido presentado como proyecto de ley estatutaria. 
Primero se expondrán las diferentes maneras en que ha sido entendida la noción enfoque diferencial por documentos y agencias oficiales. Dado que no existe un juicio único respecto a su significado, se utiliza la noción y no el concepto para enunciar el enfoque diferenciado. Luego se analiza el alcance jurídico del enfoque diferencial en el capítulo étnico del acuerdo de paz y se examina si la inserción de esta noción ha traído un mayor nivel de protección jurídica a los derechos de los grupos étnicos. Posteriormente, se exponen los efectos que puede tener el uso del enfoque diferencial en la construcción de paz desde la perspectiva de los pueblos étnicos y se analiza su interseccionalidad con un tema que aún no está resuelto en Colombia, esto es, el deber del Estado de consultar con los pueblos indígenas y otras minorías étnicas. Finalmente, se traen a la luz los desafíos que el Estado debe superar respecto a la certificación de identidad étnica, de tal suerte que se garanticen efectivamente los derechos humanos de los grupos étnicos.

\section{LA NOCIÓN DE ENFOQUE DIFERENCIAL}

El gobierno colombiano y su contraparte — las FARC-EP— afirman que el acuerdo de paz está permeado por un enfoque de derechos con el fin de que las medidas previstas "contribuyan a la materialización de los derechos constitucionales" de la población colombiana (Presidencia de la República, 2016, p. 6). En su introducción al documento, indican que la implementación del acuerdo debe "regirse por el reconocimiento de la igualdad y protección del pluralismo sin discriminación" (p. 6). Asimismo, esperan que el acuerdo genere "condiciones para que la igualdad sea real y efectiva, y se adopten medidas afirmativas a favor de grupos discriminados o marginados teniendo en cuenta el enfoque territorial, diferencial y de género" (p. 6).

Las partes mencionan, a lo largo del acuerdo, la importancia de la implementación de medidas con un enfoque territorial, diferencial y/o de género. En algunos párrafos se menciona la implementación de un enfoque territorial, en otros un enfoque diferencial o de género. Sin embargo, en ninguno de sus capítulos existe una definición sobre qué tipos de medidas o perspectivas abarca el llamado enfoque diferencial. En los principios orientadores del acuerdo está el "enfoque territorial y diferencial", pero tampoco hay una definición del significado de esa expresión. El texto se limita a expresar que las medidas de seguridad adoptadas en el marco de lo acordado deberán tener un "enfoque territorial y diferencial que tenga en cuenta las diferentes amenazas, particularidades y experiencias de las personas en su diversidad, de las comunidades y los territorios" (Presidencia de la República, 2016, p. 79).

El antes llamado Ministerio de la Protección Social (2011) entendía el enfoque diferencial como la reivindicación de una ciudadanía diferenciada "desde el reconocimiento y la redistribución, hasta la libre escogencia de llevar el tipo de vida de acuerdo a sus preferencias y capacidades" (p. 29). El Ministerio añadía que se trataba del "derecho a ejercer una ciudadanía desde la diferencia en escenarios de una 
democracia participativa, de inclusión igualitaria de ciudadanos en la escena política y en la toma de decisiones en la esfera íntima, privada y pública" (p. 29). Por otro lado, el Alto Comisionado de las Naciones Unidas para los Refugiados ha indicado que ese enfoque diferenciado implica "identificar los vacíos y riesgos de protección de cada grupo y desarrollar herramientas para dar soluciones, promover la participación equitativa, planear y ejecutar medidas afirmativas basadas en caracterizaciones sistemáticas para la garantía del goce efectivo de los derechos de los diferentes grupos poblacionales" (Naciones Unidas, 2011, p. 27). El Departamento de Prosperidad Social (s. f.) ha definido el enfoque diferencial como "el instrumento jurídico idóneo para revertir o evitar los procesos y actos que generan las condiciones actuales o históricas de discriminación e impiden el goce efectivo de derechos" (p. 1).

Como medio para ejecución de medida afirmativa para promover la participación y el ejercicio de los derechos de diferentes grupos poblacionales, el enfoque diferencial es presentado como un instrumento de protección de la discriminación racial estructural. La Convención Internacional sobre la Eliminación de todas las Formas de Discriminación Racial proclamada por la Asamblea General de Naciones Unidas (1965) prevé la implementación de medidas especiales en seguimiento de artículo 1, n. $^{\circ} 4^{2}$. Estas medidas deben ser especiales, temporales y "destinadas a garantizar el disfrute pleno e igual de los derechos humanos y las libertades fundamentales por los grupos desfavorecidos" (Naciones Unidas, 2009, párr. 11). Según el Comité de las Naciones Unidas para la Discriminación Racial —CERD—, es importante resaltar que esas medidas especiales no deben ser confundidas con los derechos permanentes que son reconocidos en los instrumentos de derechos humanos. Esto quiere decir que estas deben ser medidas que faciliten y promuevan la realización de los derechos humanos reconocidos a los grupos históricamente discriminados, pero no deben confundirse con esos derechos humanos. Las medidas especiales son provisorias y coyunturales, los derechos humanos no.

Así, desde una perspectiva jurídica, el enfoque diferencial plasmado en el acuerdo no puede ser más que una respuesta del Estado colombiano a las obligaciones asumidas que dispone la Convención Internacional para la Eliminación de todas las Formas de Discriminación Racial. El Estado debe, entonces, generar condiciones que faciliten a las poblaciones más vulnerables un nivel de igualdad sustantiva con el resto de la población, para evitar utilizar el término discriminación positiva, tal como lo recomendó el Comité en la Recomendación General número 32 del 28 de agosto de 2009:

2 En este se indica que "[llas medidas especiales adoptadas con el fin exclusivo de asegurar el adecuado progreso de ciertos grupos raciales o étnicos o de ciertas personas que requieran la protección que pueda ser necesaria con objeto de garantizarles, en condiciones de igualdad, el disfrute o ejercicio de los derechos humanos y de las libertades fundamentales no se considerarán como medidas de discriminación racial, siempre que no conduzcan, como consecuencia, al mantenimiento de derechos distintos para los diferentes grupos raciales y que no se mantengan en vigor después de alcanzados los objetivos para los cuales se tomaron" (Asamblea General de Naciones Unidas, 1965, art. 1, n.․ 4) . 
El término "medidas especiales" comprende medidas que en algunos países podrían denominarse "acción afirmativa", "medidas afirmativas" o "acción positiva" cuando corresponden a las disposiciones del párrafo 4 del artículo 1 y del párrafo 2 del artículo 2 de la Convención, como se explica en los siguientes párrafos. Al igual que la Convención, la presente recomendación emplea los términos "medidas especiales" y "medidas especiales y concretas" y alienta a los Estados partes a emplear una terminología que refleje claramente la relación entre sus leyes y prácticas y estos conceptos enunciados en la Convención. El término "discriminación positiva" es contradictorio en el contexto de las normas internacionales de derechos humanos, y debe evitarse. (Naciones Unidas, 2009, p. 4)

Ese entendimiento es coherente con la perspectiva de la Unidad de Atención y Reparación de Víctimas de la Presidencia de la República —Uariv— (s. f.), que ha incluido el enfoque diferencial étnico en las medidas de asistencia, reparación y atención integral de las víctimas del conflicto armado (p. 3). Según la Uariv (s. f.), el enfoque diferencial es un conjunto de medidas que "al dar un trato desigual o diferenciado a algunos grupos poblacionales, garantizan la igualdad en el acceso a las oportunidades sociales" (p. 4). El trato diferenciado estaría justificado por las condiciones fácticas de sus destinatarios, personas que por distintas razones históricas experimentan barreras que limitan su integración en la sociedad. La finalidad de esas medidas, según la Uariv, es hacer que esas personas puedan "acceder en condiciones de igualdad a los derechos, bienes, servicios y oportunidades sociales, compensar injusticias del pasado" (p. 4).

El Comité de Naciones Unidas para la Eliminación de la Discriminación Racial indica cuatro condiciones de aplicación de esas medidas: (1) adecuadas a la situación que quiere remediarse, (2) legítimas (3) necesarias en una sociedad democráticas y (4) respetar los principios de justicia y proporcionalidad (Naciones Unidas, 2009, párr. 16). Además, el Comité indica que las necesidades de esas medidas deben ser evaluadas sobre la base de "datos precisos, desglosados por raza, color, linaje y origen étnico o nacional y que incorporen una perspectiva de género" (Naciones Unidas, 2009, párrs. 17-18), asegurándose que las comunidades beneficiarias sean consultadas respecto a su aplicación.

En el acápite siguiente se analizarán las medidas diferenciales establecidas por el acuerdo en el capítulo étnico, con el fin de identificar su armonía con lo establecido por el CEDR de Naciones Unidas.

\section{EL ALCANCE JURÍDICO DEL ENFOQUE DIFERENCIAL EN EL CAPÍTULO ÉTNICO}

Aunque no exista consenso para definir claramente cuáles colectivos se constituyen como minorías étnicas, existen algunos factores, además de la subjetividad de los miembros, que sirven para corroborar tal condición en una determinada población. En 1977, el entonces relator especial de la Subcomisión de Prevención de Discriminaciones y Protección a las Minorías de las Naciones Unidas, definió a las minorías étnicas de la siguiente manera: 
Un grupo numéricamente inferior al resto de la población de un Estado, que se encuentra en una posición no dominante y cuyos miembros, que son nacionales del Estado, poseen características étnicas, religiosas o lingüísticas diferentes de las del resto de la población y manifiestan, aunque sólo sea implícitamente, un sentimiento de solidaridad para preservar su cultura, sus tradiciones, su religión o su idioma. (Naciones Unidas, 2010, p. 3)

En el territorio colombiano existen un total de 1.656 grupos reconocidos como minorías étnicas: 1.568 comunidades afrodescendientes reconocidas por el Ministerio del Interior en el año 2018, ochenta y siete pueblos indígenas localizados en setecientos diez resguardos y 1 pueblo rrom establecido en diecinueve ciudades de Colombia, según datos del DANE (2007).

Pese a que inicialmente el acuerdo mencionó el enfoque territorial, diferencial y de género, introdujo el enfoque étnico en el capítulo étnico. Se trata de un enfoque diferencial aplicado a la cuestión étnica. El capítulo étnico contiene una salvaguarda de protección y respeto a los estándares internacionales en el cumplimiento del deber del Estado de consultar con los pueblos étnicos.

En el capítulo étnico, el acuerdo también recalca el respeto a los derechos adquiridos y se crean algunos mecanismos para la inclusión de los pueblos étnicos como beneficiarios de las medidas de acceso a tierras (Reforma Rural Integral). El acuerdo menciona la necesidad de que las implementaciones de planes de desarrollo territorial cuenten con un mecanismo de consulta, tal como exige el derecho nacional e internacional. Asimismo, recoge el lenguaje de los derechos de los pueblos indígenas sobre sus territorios e indica que "la función ecológica de la propiedad y las formas propias y ancestrales de relacionamiento con el territorio se anteponen a la noción de inexplotación" (Presidencia de la República, 2016, p. 207).

El acuerdo garantiza la participación plena y efectiva de representantes de los pueblos étnicos en las distintas instancias que sean creadas en virtud de su implementación. Resalta la adopción de medidas que garanticen la inclusión de candidatos de los pueblos étnicos en las listas de las circunscripciones territoriales especiales de paz cuando estas coincidan con territorios de esos pueblos.

En lo que atañe a las garantías de seguridad para las comunidades étnicas, se encuentran medidas orientadas a fortalecer los sistemas de seguridad de los pueblos indígenas bajo las directrices de organismos propios, otorgando reconocimiento a la guardia indígena y cimarrona. Además, la administración de una justicia transicional debe respetar el ejercicio de las funciones jurisdiccionales de las autoridades indígenas tradicionales para dar cumplimiento a los acuerdos de paz (Presidencia de la República, 2016, p. 208). 
Es de anotar que todos esos deberes estatales supra ya estaban establecidos en los instrumentos normativos que regulan la materia en Colombia y en el sistema internacional de derechos humanos ${ }^{3}$.

El acuerdo reconoce que la persistencia de los cultivos ilícitos se debe tanto a condiciones de pobreza y de falta de políticas del Estado, como a la presencia de organizaciones criminales y violentas que se dedican al tráfico (Presidencia de la República, 2016). En este sentido, determina la necesidad de realizar políticas públicas con enfoque diferencial y de género partiendo de la premisa de que el consumo de drogas es un problema de salud pública. También se contempla la necesidad de diálogo entre todos los sectores de la población, incluyendo a las comunidades étnicas, para la elaboración de estrategias que afronten efectivamente el problema de las drogas ilícitas (Presidencia de la República, 2016). En lo relativo a los pueblos indígenas, se indica que las políticas públicas orientadas al problema de las drogas deben tener en cuenta lo siguiente:

El reconocimiento de los usos ancestrales y tradicionales de la hoja de coca, como parte de la identidad cultural de la comunidad indígena y la posibilidad de la utilización de cultivos de uso ilícito, para fines médicos y científicos y otros usos lícitos que se establezcan. (Presidencia de la República, 2016, p. 100)

El reconocimiento y la protección de las prácticas medicinales ancestrales no es una creación original del acuerdo, sino que fue recogido de lo plasmado en tratados ratificados por Colombia y sus interpretaciones oficiales. Respecto al tratamiento prestado a las víctimas del conflicto, el acuerdo se limita a reconocer la articulación del Sistema integral de Verdad, Justicia y Reparación con las funciones jurisdiccionales de las autoridades tradicionales.

En realidad no existe ninguna medida establecida como especial en los términos de la Observación General 34 del CEDR que pudiese clasificarse como una medida jurídica de enfoque diferencial, puesto que ninguna de ellas es temporal. O más bien, todas lo son, pero en función de la temporalidad de las medidas del acuerdo. Todo el capítulo étnico no hace más que reconocer los derechos humanos que, como grupos étnicos, esos pueblos ya tenían antes de la firma del acuerdo. El uso de la expresión enfoque diferencial para la implementación del capítulo étnico es equivocado y debería ser evitado por los operadores políticos y de justicia. En este sentido, es necesario recordar que:

3 En el Convenio 169 de la OIT (2015) se establece: "En la medida en que ello sea compatible con el sistema jurídico nacional y con los derechos humanos internacionalmente reconocidos, deberán respetarse los métodos a los que los pueblos interesados recurren tradicionalmente para la represión de los delitos cometidos por sus miembros. 2. Las autoridades y los tribunales llamados a pronunciarse sobre cuestiones penales deberán tener en cuenta las costumbres de dichos pueblos en la materia" (OIT, 1989, art. 9). 
Los Estados deben observar meticulosamente, en su legislación y en su práctica, la distinción entre las medidas especiales y los derechos humanos permanentes. Esta distinción implica que los titulares de esos derechos permanentes pueden beneficiarse también de las medidas especiales. (Naciones Unidas, 2009, p. 15)

De lo revisado hasta aquí, se tiene que los acuerdos de paz no han traído innovaciones en términos de reconocer nuevas garantías a las comunidades étnicas, sino que su mérito está en el campo de lo simbólico y político, puesto que se trata de una negociación entre dos actores cuyo conflicto ha mermado enormemente la calidad de vida de esas poblaciones. El documento final para la terminación del conflicto viene a decir entonces: ambas partes, Estado y FARC-EP, estamos dispuestas a trabajar juntos para que los grupos étnicos puedan ejercer plenamente sus derechos. Ese es el verdadero valor de lo acordado en la Habana.

\section{EL ENFOQUE DIFERENCIAL EN LA CONSTRUCCIÓN DE LA PAZ}

La paz es un tema lejano para muchas poblaciones situadas en las regiones del interior de Colombia, donde la violencia ha llegado a convertirse en el motor de la economía que moldea el pensamiento de quienes han tenido que vivir en medio del conflicto. Por ello, existe la inquietud respecto a cuál es el concepto de paz que ofrecen los acuerdos de La Habana: si se trata de una paz construida a partir de políticas públicas diseñadas por los agentes burocráticos que no han vivido los efectos de una guerra, o si efectivamente se construirá una paz de forma articulada con quienes han sufrido la violencia en sus cuerpos, familias y comunidades.

Teniendo en cuenta que la concepción de paz de las Naciones Unidas no se limita al cese de la violencia armada dentro de un Estado o entre dos o más Estados, sino que representa una amplia protección de los derechos humanos y el respeto por la diversidad, se puede afirmar que la paz no se logra pensando por los otros. La paz no se traduce únicamente en un cese de hostilidades, sino en la posibilidad de que un pueblo o una comunidad pueda efectivamente construir su visión de futuro. Esto no solo representa un reto para quienes firmaron la paz, sino para toda la sociedad colombiana, puesto que sienta las bases para un diálogo donde se expongan los intereses que existan en común y aquellos aspectos en los cuales hay tensiones y diferencias, con el objetivo de generar propuestas que favorezcan a todos.

El capítulo étnico del acuerdo de paz fue el resultado de un proceso concertado entre organizaciones indígenas y comunidades afrodescendientes. Si bien el documento resultante de la mesa de concertación no fue incluido en su totalidad, significa un avance en las negociaciones entre el Gobierno colombiano y los grupos étnicos. La incorporación de algunos de los parámetros de protección de los derechos de las minorías en el acuerdo de paz, indica el reconocimiento de estos como mecanismos adecuados para implementar procesos de justicia. Teniendo en 
cuenta que las Naciones Unidas y la comunidad internacional han jugado un papel relevante para el éxito de la firma del acuerdo final, es fundamental que las medidas que de ahí emanen se encuentren alineadas con el derecho internacional de los derechos humanos.

Sin embargo, lo que preocupa a muchos líderes e intelectuales del movimiento indígena colombiano respecto a la implementación de lo acordado es la poca voluntad de los sucesivos gobiernos para implementar métodos idóneos de consulta con las comunidades tradicionales (M. Mazabel, comunicación personal, 18 de abril de $2018)^{4}$. Si la consulta ha sido celebrada en el acuerdo como uno de los mecanismos esenciales para el diálogo y para la construcción de paz, es necesario preguntarse cómo el gobierno colombiano ha entendido y entiende la consulta.

La Directiva Presidencial 01 (2010), de autoría del expresidente Uribe Vélez es la normativa que el Ministerio del Interior entiende como vigente, pese a que esta sufre de por lo menos tres problemas jurídicos graves: (1) no fue consultada con los pueblos indígenas, requisito indispensable para su vigencia según la Ley $21^{5}$ (1991) que acogió el Convenio 169 de la OIT en Colombia; (2) aparentemente el objetivo de tal Directiva fue eximir ciertas actividades del proceso de consulta, como establecer la imposibilidad de que las comunidades consultadas puedan vetar cualquier propuesta $^{6}$; (3) una directiva presidencial no puede regular una materia de derechos humanos que, por su naturaleza sustantiva, debe ser regulada por ley estatutaria.

La Directiva Presidencial 01 (2010) es un buen ejemplo de la confusión que el Gobierno nacional y otras instancias estatales tienen respecto al significado del enfoque diferencial. La Directiva prevé su aplicación, entre otros, "cuando se

4 La Directiva Presidencial 01 (2010) indica que "la consulta procede antes de la ejecución o puesta en marcha de cualquier proyecto que pueda afectar a los Grupos Étnicos Nacionales, o los derechos de los que son titulares de Acuerdo con la Declaración Universal de Derechos Humanos, el Pacto Internacional de Derechos Económicos, Sociales y Culturales, el Pacto Internacional de Derechos Civiles y Políticos, o los Instrumentos internacionales sobre la prevención de la discriminación. En este sentido se relacionan las siguientes acciones que deben consultarse con los mencionados Grupos: Formulación, diseño o ejecución de proyectos de investigación adelantados por Entidades Públicas que estén relacionados con los recursos naturales, bióticos, económicos, culturales, religiosos, etc., de los grupos étnicos y puedan generar una afectación por la ejecución o la publicación de los mismos" (p. 3).

5 En esta ley se establece: "Al aplicar las disposiciones del presente Convenio, los gobiernos deberán: a) Consultar a los pueblos interesados, mediante procedimientos apropiados y en particular a través de sus instituciones representativas, cada vez que se prevean medidas legislativas o administrativas susceptibles de afectarles directamente" (Ley 21, 1991, art. 6.1).

6 "Si bien es cierto que la realización del proceso de Consulta Previa en los casos previstos en los acuerdos internacionales es obligatoria, los Grupos Étnicos Nacionales, en ejercicio de este derecho fundamental, no pueden vetar el desarrollo de proyectos" (Directiva Presidencial 01, 2010).

7 En la Constitución Política (1991) se establece: "Mediante las leyes estatutarias, el Congreso de la República regulará las siguientes materias: a) Derechos y deberes fundamentales de las personas y los procedimientos y recursos para su protección" (art. 152). 
expidan medidas legislativas o administrativas susceptibles de afectar directa y específicamente a los Grupos Étnicos Nacionales, y que requieran en el ámbito de su aplicación la formulación de enfoque diferencial" (art. 2, énfasis añadido). De hecho, la consulta previa debe ser realizada cada vez que esas medidas — legislativas o administrativas - afecten a los pueblos interesados, y no solamente en la aplicación de medidas específicas (Sentencia C317, 2012).

En el año 2017, el Poder Ejecutivo presentó al Congreso un Proyecto de Ley de consulta que no dejaba espacio para que los grupos consultados incidieran en las cuestiones sustantivas de las decisiones, lo que significa un tipo de consulta que en algunos puntos va en contra lo establecido en el derecho internacional, como poner limitación de los asuntos a ser consultados, exentando de esta, por ejemplo, leyes y actos administrativos que pretendan garantizar el orden público y la seguridad (Ministerio del Interior, 2017). Esa propuesta fue archivada, y en septiembre de $2018 \mathrm{el}$ partido político Cambio Radical presentó ante el Senado de la República el Proyecto de Ley 134 (2018) que pretendía regular el derecho fundamental a la consulta previa.

Este proyecto generó preocupación porque respondía a motivos distintos a los expresados en la firma del acuerdo de paz. Según sus proponentes, se trataba de un esfuerzo de varios años entre los actores del sector público y privado para regular ese derecho. El proyecto, sin embargo, no buscaba conciliación o justicia histórica como promete el acuerdo, sino que, por el contrario, partía de la desconfianza sobre la efectividad de la herramienta de consulta y anunciaba un esfuerzo para limitar su alcance, tal como se evidenciaba en la exposición de motivos:

Cabe destacar que en Colombia, con una población indígena que no supera en 3,5 por ciento del total de habitantes, se han ejecutado aproximadamente más de 4.500 procesos de consulta previa, mientras que en Chile, Perú, Bolivia, con poblaciones indígenas mucho mayores, han realizado 36, 28 y 40, respectivamente. Incluso se trata de naciones con recursos, tradición y actividad minera más importantes que los nuestros. ¿Qué puede explicar esta situación? Podría decirse que obedece a una interpretación excesivamente garantista de la Constitución y/o un posible abuso de quienes intervienen en estas. (Proyecto de Ley 134, 2018, p. 20)

Por otro lado, se decía que el impacto de la medida a implementar debía ser "determinado o determinable y siempre verificable por parte de la Dirección de Consulta Previa del Ministerio del Interior" (Proyecto de Ley 134, 2018, p. 23). Pero como se expondrá más adelante en este trabajo, en ocasiones esa función de análisis de campo por parte de la Dirección termina siendo un proceso formal que no garantiza la verdadera protección de un derecho fundamental como lo es la consulta previa, libre e informada. Asimismo, la exposición de motivos del Proyecto de Ley resaltaba que la consulta previa, "al no ser un derecho absoluto, no puede constituir una especie de veto en la toma de decisión legítima por parte del Estado respecto de la ejecución de la medida" (Proyecto de Ley, 2018, p. 35.), por lo que en caso de que no se lograra un 
consenso entre proponentes y comunidades étnicas, el Estado es quien determinaría la ejecución o no de esta. En este sentido, la propuesta imponía un plazo final y tajante para que dejara de existir "una indefinición al respecto" a la viabilidad del proyecto.

Aunque la consulta, tal como el derecho a la vida, a la propiedad, y a la libertad de expresión y muchos otros derechos fundamentales, no sea un derecho absoluto, ello no significa que el gobierno de un Estado tenga un cheque en blanco respecto a la ejecución de una medida que pueda afectar a una determinada comunidad. La jurisprudencia de la Corte Interamericana de Derechos Humanos (2006) y también la de la Corte Constitucional de Colombia (2018) han indicado que cuando proyectos de desarrollo a gran escala puedan afectar territorios tradicionales, el Estado tiene el deber de obtener el consentimiento libre, previo e informado de las comunidades.

Esa caracterización de no veto que imponía el proyecto al resultado de la consulta, no indica solamente el desconocimiento de la jurisprudencia sobre el tema, sino que merma el proceso desde sus inicios, lo que le da el mensaje al pueblo consultado de que, sin importar el resultado de la consulta, el gobierno tomará la decisión que le complazca. En ese mismo sentido, causa preocupación que sectores del Legislativo insistan en excluir del proceso de consulta las medidas relacionadas con actividades de la Fuerza Pública ${ }^{8}$. Si bien es cierto que para la defensa de la soberanía, la integridad del territorio y el mantenimiento de la paz, sería contraproducente pedir permiso para entrar en un determinado espacio geográfico, sí deben existir acuerdos y negociaciones para la instalación de bases militares u operativos de rutina en territorios indígenas, especialmente en tiempos de posacuerdo.

Al analizar otro punto del Proyecto de Ley 137, se debe destacar la intención para desestimar la consulta previa en aquellos proyectos que ya están en desarrollo y que no fueron previamente consultados ${ }^{9}$, cuestión que no solo viola el deber del Estado colombiano de consultar, sino que además choca con la posición de la Corte Constitucional:

Cuando ya se ha iniciado la implementación del proyecto, la obligación de consulta persiste y pese a su omisión no se invalida, pues se trata de la vulneración de un derecho humano fundamental cuya afectación es continua en el tiempo. Adicionalmente, en caso de existir un cambio sustancial en las condiciones del proyecto, que implique la adopción de nuevas medidas o la alteración del significado concreto de medidas ya tomadas, el deber de consulta se renueva pese a que

8 El Proyecto de Ley 137 (2018) indicaba lo siguiente: "Medidas que no requieren de consulta previa. No requieren de consulta previa: (V) Las actividades desarrolladas por la Fuerza Pública en uso exclusivo de sus competencias" (art. 50).

9 El Proyecto de Ley 137 (2018) proponía lo siguiente: "Invalidez de los Procesos de Consulta Previa. No pueden entenderse como válidos los procesos de consulta previa que: (ii) los procesos consultivos realizados con posterioridad a la implementación, ejecución o consumación de medidas que han debido ser consultadas previamente" (art. 49). 
el proyecto se encuentre en desarrollo. En estas circunstancias, la jurisprudencia ha indicado que esta obligación exige la identificación de las nuevas afectaciones que surjan en la realización de la actividad, al igual que en las fases restantes del proyecto.

Cuando el proyecto ha finalizado, la consulta se dirige a buscar los remedios para reparar, recomponer y restaurar la afectación al tejido cultural, social, económico o ambiental, los cuales, deben responder a la clase de daño sufrido por la comunidad étnica. Tal regla tiene fundamento no solo en el principio general del derecho según el cual todo daño antijurídico debe ser reparado, sino porque el juez constitucional no puede avalar la vulneración de los derechos fundamentales o declarar la ocurrencia de un daño consumado en materia de consulta previa, pues se crearía un incentivo indebido para evadir esta obligación constitucional (Sentencia SU-123, 2018).

En general, toda la propuesta de ley iba en contra del principio de la buena fe en el Derecho Internacional Público y desconocía la jurisprudencia internacional y doméstica sobre la materia. Aunque la propuesta fue archivada, es muy importante anotar sus falencias porque el proyecto tendía a recoger las principales tensiones respecto a la consulta previa que han existido entre gobiernos de turno y poblaciones afectadas por proyectos extractivos, y es bastante probable que una propuesta similar a esa resurja en el debate legislativo.

En ese mismo sentido, se debe resaltar que, el artículo 6 del Convenio 169 de la OIT (1989) es muy claro en determinar que los Estados deben consultar primero y coordinar con los grupos étnicos el instrumento de consulta a ser implementado. Esto quiere decir que los detalles del procedimiento de la consulta previa deben ser concertados con los pueblos a ser consultados, antes de la implementación de la consulta. Teniendo en cuenta que el proyecto fue elaborado entre el Gobierno y empresas privadas a espaldas del movimiento indígena, es incomprensible que el Senado colombiano se haya auto atribuido legitimidad para proponer una ley de regulación de la consulta previa.

Aunado a lo anterior, se tiene que el partido que elaboró y presentó el Proyecto de Ley 134 (2018) manifestó en la exposición de motivos que una interpretación excesivamente garantista de la Constitución ha llevado a que en el país exista un desbordado uso de la herramienta de consulta, comparado con países como Chile, Perú o Bolivia (Sentencia SU095, 2018). Esa perspectiva deja visible que la preocupación central de los legisladores proponentes está mucho más alineada con una visión utilitarista del Estado antes que la protección y promoción de los derechos humanos de todos los colombianos. De cualquier forma, mientras ningún proyecto sea consultado y concertado con las organizaciones y comunidades destinatarias, no encontrará legitimidad. Asimismo, las organizaciones afros e indígenas, por medio de sus representantes, han indicado al Gobierno que no desean la implementación de una ley nacional de consulta, sino que es necesaria la implementación de protocolos específicos de 
consulta con cada comunidad, que den cuenta de la diversidad de pueblos, intereses y costumbres (M. Mazabel, comunicación personal, 18 de abril de 2018).

\section{CERTIFICACIÓN DE IDENTIDAD}

Otro tema tratado en la propuesta de ley de consulta fue la "certificación" de los pueblos indígenas, afrocolombianos, rrom, palenqueros y raizales, evidenciando la pretensión de creación de un Registro Único de Pueblos (RUP) con el fin de listar a los pueblos que habitan en un determinado lugar del territorio colombiano y que en algún momento podrían ser objetos de consulta. Lo peligroso de ello es la pretensión del Estado de adjudicarse la potestad de determinar la identidad, y por ende, la subjetividad de una persona o grupo de personas. Ello significaría imponer un obstáculo al ejercicio de un derecho fundamental, cuestión que es incompatible con la Constitución colombiana y el derecho internacional de los derechos humanos. Desde una perspectiva antropológica, no es posible certificar la identidad de nadie. Tal como lo expone Viveiros de Castro (2018), los enunciados de indianidad (y, agregamos, de cualquier otra identidad) son enunciados performativos y no constatables, por ende, no son certificables ${ }^{10}$. La Corte Constitucional colombiana llegó a la misma conclusión cuando decidió que el deber de consultar surge de la titularidad de derechos y no de los registros del Estado" ${ }^{11}$.

La aplicación de un mecanismo de consulta no puede cimentarse sobre la idea de la certificación de identidades, puesto que se entraría a cosificar el grupo y lo convertiría en un dato digital que, de no estar en las planillas de las subsecretarías en Bogotá, sencillamente no existiría. De hecho, uno de los temas que más nerviosismo genera entre el movimiento indígena y las comunidades afros es la expedición de certificaciones que reconozcan o nieguen la existencia de comunidades en el territorio colombiano (M. Mazabel, día de julio, 2018). Esto obvia que "la jurisprudencia ha precisado que la ausencia de reconocimiento oficial de una comunidad es insuficiente

10 Según Viveiros de Castro (2018): "Los enunciados de indianiedad son enunciados performativos y no enunciados constatativos, dependiendo, por lo tanto, de condiciones de felicidad y no de condiciones de verdad (en el sentido de corresponder con un estado de cosas). Empero, y este es el punto, las condiciones antropológicas de felicidad de tal enunciado no son dadas por terceros. Sobre todo, no son ni pueden ser dadas por el Estado, el Tercero por excelencia. La identidad es tautegórica; ella crea su propia referencia. Indios son aquellos que "representan a sí mismos" (p. 194).

11 "La obligación de consultar la ejecución de un proyecto con la comunidad étnicamente diferenciada surge de la titularidad de los derechos derivados de la identidad étnica diversa. Esa calidad se origina en factores objetivos así como subjetivos, y no deriva de registros del Estado. La certificación de la presencia de colectividades étnicas es una medida que racionaliza la actuación de la administración y de los particulares, empero carece de la idoneidad para demostrar la presencia de esos grupos étnicos, al punto que la realidad prevalecerá cuando a esa constatación formal no obedece a aquella. En atención a dicho criterio, diferentes Salas de Revisión han descartado las certificaciones de existencia de comunidades indígenas o tribales, en el evento en que esa verificación es insuficiente frente a la presencia de la colectividad o no comprenda las realidades sociales, económicas, espirituales y rituales que implica una concepción amplia de territorio" (Sentencia SU-123, 2018). 
para que el Estado o un privado se niegue a consultar una medida" (Sentencia SU-123, 2018). Esta situación ha provocado la vulneración de los derechos fundamentales de muchos pueblos, so pretexto de su inexistencia ante los ojos del Estado. La Corte Constitucional ha dicho recientemente que la certificación de la existencia o no de un grupo étnico en un determinado territorio no suple los estudios que se deben hacer respecto de la vulneración que un proyecto pueda traer a los grupos étnicos. Así, añade la Corte, "la consulta es obligatoria cuando, pese a la certificación de ausencia de colectividad, se verifica la afectación" (Sentencia SU-123, 2018, p. 81).

La propuesta de Proyecto de Ley 134 no favoreció la construcción del clima de confianza necesario entre el Gobierno y los pueblos indígenas para el fortalecimiento y mantenimiento de la paz. Por el contrario, esa propuesta fue en contra de lo determinado por el acuerdo de paz al poner en duda uno de los principales mecanismos de implementación de la paz, esto es, la consulta previa, libre, informada y de buena fe.

\section{CONCLUSIONES}

Las disposiciones contenidas en la propuesta de ley estudiada de forma precedente resultaban bastante nocivas para las comunidades étnicas en Colombia, por cuanto proponían una relación tutelar e impositiva entre el Estado colombiano y los pueblos tradicionales. Teniendo en cuenta que el capítulo étnico del acuerdo de paz se orienta por los mecanismos de consulta, el florecimiento de esa iniciativa hubiese puesto en riesgo todas las intenciones plasmadas en el acuerdo en lo relacionado con los pueblos indígenas, afrocolombianos y rrom. El fracasado proyecto de ley de consulta muestra que aún hay mucho que aprender y avanzar respecto a la ética en la política: el enfoque diferencial debe significar una verdadera herramienta de oportunidad para la construcción de igualdad sustantiva, y no un término vacío de contenido que confunda a la población y distraiga la atención sobre los derechos de los pueblos étnicos. En otras palabras, si el enfoque diferencial no es una medida afirmativa en los términos que propone el Comité para la Eliminación de la Discriminación Racial de las Naciones Unidas, no tiene contenido jurídico y su uso debe ser evitado en el ámbito del derecho.

Pese a lo establecido en el acuerdo de paz respecto a las comunidades étnicas, la realidad dista mucho de lo pactado, puesto que la actitud del Legislativo sigue siendo la misma frente a temas de crucial importancia como lo es la consulta previa. Hay apatía y desinterés por tomar en consideración la postura de quienes se ven directamente afectados por el desarrollo de un proyecto, o la aplicación de una medida administrativa o legislativa.

El éxito de la implementación del acuerdo de paz residirá en la capacidad de los gobiernos de abrir canales de participación con las comunidades afectadas y respetar su voz, pues esa es la única manera de construir una paz que vaya de la mano con lo 
propuesto por las Naciones Unidas: una paz que no signifique solamente la ausencia de conflicto armado, sino que fortalezca la voz y dé lugar a la visión de las comunidades afros, indígenas, raizales y rrom, especialmente en aquellos casos en que estos pueblos se enfrenten con proyectos de gran infraestructura energética, tales como los que están siendo planteados para la Colombia del posacuerdo.

\section{REFERENCIAS}

Asamblea General de Naciones Unidas. (1965, 21 de diciembre). Convención Internacional sobre la Eliminación de todas las Formas de Discriminación Racial. Resolución 2106 A (XX). https://www.ohchr.org/ SP/ProfessionalInterest/Pages/CERD.aspx

Asamblea General de Naciones Unidas. (2011). Informe sobre la marcha de los trabajos del Comité Asesor del Consejo de Derechos Humanos sobre el derecho de los pueblos a la paz. A/HRC/17/39. https://www.ohchr. org/Documents/HRBodies/HRCouncil/RegularSession/Session20/A.HRC.20.31.SPA.pdf

Asamblea Nacional Constituyente. (1991). Constitución Política de Colombia. http://www.secretariasenado. gov.co/senado/basedoc/constitucion_politica_1991.html

Bustos, C. y Jaramillo, M. (2016, 24 de octubre). ¿Quétiene que ver con el medio ambiente la paz en Colombia? The Guardian. https://www.theguardian.com/global-development-professionals-network/2016/ oct/24/medio-ambiente-paz-colombia

Congreso de Colombia. (1991, 4 de marzo). Ley 21 de 1991. Por medio de la cual se aprueba el Convenio número 169 sobre pueblos indígenas y tribales en países independientes, adoptado por la 76a reunión de la Conferencia General de la OIT, Ginebra 1989. Diario Oficial n. ${ }^{\circ}$ 39729. http://www.suin-juriscol.gov.co/ viewDocument.asp?ruta=Leyes $/ 1577376$

Congreso de la República de Colombia. (2018). Proyecto de Ley 134 de 2018. Por el cual se regula el Derecho Fundamental a la Consulta Previa y se dictan otras disposiciones. https://docs.google.com/viewerng/ viewer?url=http://leyes.senado.gov.co/proyectos/images/documentos/Textos+Radicados/ proyectos+de+ley/2018+-+2019/PL+134-18+Consulta+Previa.pdf

Corte Constitucional. (2012, 24 de octubre). Sentencia C317/12 (María Victoria Calle Correa, M.P.). https://www.corteconstitucional.gov.co/relatoria/2012/C-317-12.htm

Corte Constitucional de Colombia. (2018, 11 de octubre). Sentencia SU-095/2018 (Cristina Pardo Schlesinger, M.P.). https://www.corteconstitucional.gov.co/relatoria/2018/SU095-18.htm

Corte Constitucional de Colombia. (2018, 15 de noviembre). Sentencia SU123/18 (Alberto Rojas Ríos, M. P.). https://www.corteconstitucional.gov.co/relatoria/2018/SU123-18.htm

Corte Interamericana de Derechos Humanos, Corte IDH. (2006). Sentencia de 29 de marzo de 2006 para el caso Comunidad Indígena Sawhoyamaxa vs. Paraguay.

Departamento de Prosperidad Social. (s. f.). Grupo de Enfoque Diferencial. http://www.prosperidadsocial. gov.co/ent/gen/prg/Documents/Descripci\%C3\%B3n\%20Enfoque\%20Diferencial.pdf

Departamento Administrativo Nacional de Estadística, DANE. (2007). Colombia una nación multicultural. https://www.dane.gov.co/files/censo2005/etnia/sys/colombia_nacion.pdf

Gaviria, S. (2016). Por cada año de paz, Colombia ahorraría $\$ 7,1$ billones en degradación ambiental. Portal web Departamento Nacional de Planeación. https://www.dnp.gov.co/Paginas/\%E2\%80\%9CPor- 
cada-a\%C3\%Blo-de-paz,-Colombia-ahorrar\%C3\%ADa-\$7,1-billones-en-degradaci\%C3\%B3nambiental\%E2\%80\%9D-Sim\%C3\%B3n-Gaviria-Mu\%C3\%Bloz.aspx

Ministerio de la Protección Social. (2011). Directriz de enfoque diferencial para el goce efectivo de los derechos de las personas en situación de desplazamiento con discapacidad en Colombia. https://www.minsalud.gov. co/Documentos\%20y\%20Publicaciones/Directriz\%20Persona\%20en\%20Desplazamiento\%20 con\%20Discapacidad.pdf

Ministerio del Interior. (2010, 26 de marzo). Directiva Presidencial 01. Garantía del derecho fundamental a la consulta previa de los grupos étnicos nacionales. https://www.mininterior.gov.co/sites/default/files/13 directiva_presidencial_01_de_2010.pdf

Ministerio del Interior. (2017). Proyecto de Ley. Por la cual se adopta el procedimiento administrativo de consulta previa, se ordena la creación de la Unidad de Consulta Previa en el Ministerio del Interior, y se dictan otras disposiciones.

Naciones Unidas. (2009). Comité para la Eliminación de la Discriminación Racial. Recomendación General 32. https://www.acnur.org/fileadmin/Documentos/BDL/2012/8464.pdf

Naciones Unidas. (2010). Derechos de las minorías: Normas Internacionales y orientaciones para su aplicación. https://www.ohchr.org/Documents/Publications/MinorityRights_sp.pdf

Naciones Unidas. (2011) Oficina del Alto Comisionado para Refugiados. Directriz de enfoque diferencial para el goce efectivo de derechos de las personas en situación de desplazamiento forzado con discapacidad en Colombia. https://www.acnur.org/fileadmin/Documentos/BDL/2011/7536.pdf

Organización Internacional del Trabajo, OIT. (1989). Convenio 169 sobre pueblos indígenas y tribales en países independientes.

Organización Internacional del Trabajo. (2016). Reporte Regional Colombia, Costa Rica, Guatemala, Chile. Convenio 169 de la OIT sobre Pueblos Indígenas y Tribales en Países Independientes y la consulta previa a los pueblos indígenas en proyectos de inversión. https://www.ilo.org/wcmsp5/groups/public/---americas/--ro-lima/documents/publication/wcms_507556.pdf

Perald, A. (2016, 23 de junio). La paz: oportunidad para el medio ambiente en Colombia. Programa de las Naciones Unidas para el Desarrollo. https:/www.undp.org/content/undp/es/home/blog/2016/6/23/ La-paz-oportunidad-para-el-medio-ambiente-en-Colombia.html

Presidencia de la República. (2016). Acuerdo Final para la Terminacion del Conflicto y la Construcción de una Paz Estable y Duradera. http://www.altocomisionadoparalapaz.gov.co/procesos-y-conversaciones/ Documentos\%20compartidos/24-11-2016NuevoAcuerdoFinal.pdf

República de Colombia. (2018, 20 de septiembre). Gaceta del Congreso 739, Año XXVII, n.o 739. http:// www.secretariasenado.gov.co/legibus/legibus/gacetas/2018/GC_0739_2018.pdf

Semana. (2014, 29 de marzo). Pesadilla Ambiental. Revista Semana. https://www.semana.com/nacion/ articulo/sequia-en-casanare-otros-conflictos-ambientales-en-colombia/381836-3

Unidad para la Atención y Reparación Integral para las Víctimas. (s. f.). Enfoque Étnico. Pueblos y comunidades indígenas, comunidades negras, afrocolombianas, raizales, palenqueras y pueblo rrom. https:// www.unidadvictimas.gov.co/sites/default/files/documentosbiblioteca/enfoqueetnico.PDF

Vallejo, F. (2016). El proceso de consulta previa en los fallos de la Corte Constitucional de Colombia. Opinión Jurídica, 14(2).

Viveiros De Castro, E. (2018). En Brasil todos son indios, excepto quien no lo es. Jangwa Pana, 17(2). 